\title{
Sécurité des piétons dans les rues équipées de couloirs réservés aux bus
}

\section{Pedestrian safety in streets equipped with bus lanes}

\author{
Jean-Yves Fournier · Nicolas Clabaux · Thierry Brenac \\ C IFSTTAR et Éditions NecPlus 2016
}

\begin{abstract}
Résumé Cet article traite des incidences sur la sécurité des piétons de l'aménagement de couloirs de circulation réservés aux bus dans les grandes agglomérations. Après une revue de la littérature scientifique, il présente une investigation sur la sécurité des piétons dans les rues équipées de voies réservées aux bus dans la ville de Marseille. Cette investigation comporte des analyses quantitatives des risques d'accidents de piéton et une analyse qualitative du déroulement de cas d'accidents de piéton survenus dans ces voies réservées. Les conclusions issues de la littérature et les résultats obtenus sur le terrain étudié sont en bonne concordance et suggèrent globalement que les couloirs de bus, aménagés dans le sens de la circulation générale ou à contresens, sont plutôt défavorables à la sécurité des piétons. Les résultats montrent également que le type d'aménagement conditionne les formes d'accidents de piétons et donc les moyens envisageables pour leur prévention.
\end{abstract}

Mots clés voie réservée aux bus · transports publics · sécurité de la circulation · piéton · accident

\begin{abstract}
This article deals with the effects on pedestrian safety of the implementation of bus lanes in great cities. After a review of the scientific literature, an investigation into the safety of pedestrians in streets with bus lanes in the city of Marseille is presented. This investigation includes quantitative analyses of pedestrian accident risks and a qualitative analysis of pedestrian accident cases occurred in bus lanes. The conclusions drawn from the literature review
\end{abstract}

Jean-Yves Fournier $(\triangle)$

Ifsttar, TS2, LMA, F-13300 Salon de Provence, France

e-mail : jean-yves.fournier@ifsttar.fr

Nicolas Clabaux $(\square)$

Ifsttar, TS2, LMA, F-13300 Salon de Provence, France

e-mail : nicolas.clabaux@ifsttar.fr

Thierry Brenac $(\bowtie)$

Ifsttar, TS2, LMA, F-13300 Salon de Provence, France

e-mail : thierry.brenac@ifsttar.fr and the results obtained from the field investigation are consistent and, as a whole, suggest that bus lanes (with-flow or contraflow bus lanes) tend to be detrimental to the safety of pedestrians. The results also show that the various types of bus lane give rise to different forms of pedestrian accidents and therefore to different possibilities in terms of preventive measures.

Keywords bus lane $\cdot$ public transport - traffic safety · pedestrian $\cdot$ accident

\section{Introduction}

En Europe, les premiers couloirs réservés aux autobus furent introduits à titre expérimental à partir de la fin des années 1950 (en 1958 à Stockholm, en 1964 à Paris et Marseille, par exemple) pour faire face aux encombrements et à la pollution atmosphérique dans les grands centres urbains [1]. Ils font l'objet d'un regain d'intérêt, en particulier depuis le début des années 2000, en lien avec les préoccupations de développement durable. Ces dernières années, les nombreuses créations de lignes de bus à haut niveau de service ont par exemple contribué à accroître le nombre de voies réservées aux bus aménagées dans les agglomérations françaises [2].

Cette affectation d'une partie de la chaussée à la circulation des bus n'est pas sans incidence sur la sécurité des usagers de l'espace public. Divers travaux suggèrent que l'aménagement de voies réservées aux bus conduit souvent à une augmentation des nombres d'accidents corporels de la circulation (voir notamment [3] et la méta-analyse générale rapportée par Høye et al. [4]). L'existence d'incidences négatives sur la sécurité ne signifie pas qu'il faille renoncer à de tels aménagements. Elle suggère cependant qu'une meilleure connaissance des risques attachés à différentes configurations de ces voies réservées, et des processus par lesquels ces configurations peuvent contribuer à la genèse de certains accidents, serait utile pour mieux éclairer les choix d'aménagement et pour envisager les possibilités d'amélioration de la conception de ces voies. 
Nous proposons dans cet article d'examiner plus particulièrement l'effet qu'ont les couloirs réservés aux bus sur les accidents corporels touchant des piétons (quels que soient les véhicules impliqués dans ces collisions : bus, taxis, autres véhicules, autorisés ou non). Cette question a été moins étudiée. Dans un premier temps, nous passons en revue les travaux publiés sur ce sujet dans la littérature scientifique internationale (section 2). Nous verrons que, bien que les voies réservées aux bus soient des aménagements assez anciens, les connaissances concernant leurs effets sur la sécurité des piétons restent à ce jour limitées, et que sur ce sujet très peu de travaux ont été publiés concernant des villes françaises. C'est pourquoi nous proposons ensuite de contribuer à l'amélioration de ces connaissances en nous appuyant sur plusieurs analyses menées sur la ville de Marseille. Après la présentation du terrain d'étude, des données et de quelques éléments descriptifs généraux concernant les aménagements réservés aux bus dans la ville de Marseille (section 3), nous tentons d'estimer les risques d'accident de piéton attachés aux sections de rue équipées de couloirs de bus par comparaison avec des sections non équipées (section 4). La question plus spécifique du risque d'accident de piéton lié à la circulation dans les couloirs de bus par rapport à la circulation dans les files adjacentes est traitée dans la section 5. Enfin nous nous intéressons aux processus à l'œuvre dans le déroulement d'accidents de piéton survenus sur des voies réservées aux bus, par l'étude qualitative des procès-verbaux d'accidents (section 6).

\section{Voies réservées aux bus et sécurité des piétons : enseignements de la littérature internationale}

\subsection{Voies réservées aux bus dans le sens de la circulation générale}

L'effet sur la sécurité des piétons des voies réservées aux bus implantées dans le sens de la circulation générale a fait l'objet d'assez peu d'investigations. Les analyses conduites à Londres par Devenport, portant sur 33 sections urbaines avant et après la mise en service de couloirs dans le sens de la circulation, montrent que les nombres d'accidents de piéton ont significativement augmenté (d'environ $27 \%$ ) entre les deux périodes de 3 ans avant et après la mise en service, pendant les heures de fonctionnement de ces couloirs [5]. Aucune évolution significative n'est constatée en dehors des heures de fonctionnement. L'étude des accidents montre que c'est principalement du côté où la voie bus est implantée que le nombre d'accidents a augmenté. Dans la continuité de ces travaux, Brownfield et Devenport observent que le nombre moyen d'accidents de piétons recensés sur les passages piétons de 30 sections tirées aléatoirement parmi 200 sections du Grand-Londres équipées de voies bus dans le sens de la circulation, est nettement supérieur à la moyenne observée sur les passages piétons des rues du Grand-Londres (supérieur de $35 \%$ et $75 \%$, respectivement, pour les passages piétons à feux et les passages piétons de type zébra) [6]. Les auteurs ne tiennent toutefois pas compte des trafics et des mouvements de piétons, qui différent sans doute nettement entre les sections équipées de couloirs de bus, plus fréquentes dans les secteurs les plus centraux, et l'ensemble des rues du Grand-Londres. La différence observée est donc peut-être davantage le reflet de différences d'environnement de circulation et de fréquentation piétonne entre les rues équipées de couloirs et les autres rues du Grand-Londres que le révélateur d'un sur-risque. En France, concernant Paris et sa banlieue, Vayre rapporte des résultats se limitant à l'analyse du risque de choc entre bus et piéton, par kilomètre parcouru par un bus. Dans Paris intra-muros, ce risque apparait inférieur dans les couloirs de bus dans le sens de la circulation générale, par rapport aux bus circulant dans les voies de circulation ordinaires, mais le résultat contraire est obtenu en banlieue [7].

Des investigations plus récentes ont été conduites dans des pays industrialisés non européens. Dans une robuste étude avant-après portant sur la ville de New York, Chen et al. comparent le nombre d'accidents de piétons recensés sur 210 sections équipées de couloirs de bus deux ans après leur mise en service, au nombre d'accidents tel qu'il aurait dû être s'il avait suivi la même tendance que celle observée sur un groupe de sections témoins (étude quasi-expérimentale). L'étude des accidents s'appuie sur cinq années d'observation avant la mise en service et deux années après. Les résultats montrent que la création de voies réservées aux bus, reposant notamment sur la suppression des files de véhicules en stationnement, a conduit à une augmentation significative des accidents corporels de piétons $(+155 \%$ en section courante et $+33 \%$ en carrefour) [3]. Une autre étude avant-après, conduite à Melbourne (Australie) par Goh et al., évalue les effets de l'implantation de mesures donnant la priorité à des bus sur quatre itinéraires à haut niveau de service, comportant notamment la création de voies réservées dans le sens de la circulation. Les résultats ne mettent pas en évidence d'évolutions significatives des accidents de piétons entre la période avant et la période après. Une tendance à la hausse de leur fréquence et de leur gravité est observée, mais les effectifs sont trop faibles pour pouvoir conclure [8]. Notons que cette étude porte en grande partie sur des sections situées dans la périphérie de Melbourne où les mouvements de piétons sont probablement plus limités qu'en centre-ville. 
Enfin d'autres travaux ont porté sur des villes d'Amérique latine ou d'autres pays émergents. Des analyses transversales modélisant les effets de caractéristiques d'aménagement et de trafic sur les accidents, sur un ensemble d'infrastructures d'une même ville, ont été rapportées par Duduta et al. [9]. Un effet défavorable significatif des voies de bus ordinaires (dans le sens de la circulation générale) sur la sécurité des piétons est observé à Guadalajara (Mexique) mais aucun effet significatif n'est mis en évidence à Mexico. Ces auteurs rapportent d'autre part les effets de la mise en place de voies de bus en position centrale à Delhi (Inde) sur la base de données avant-après : une augmentation notable des accidents mortels de piétons a été constatée. Enfin, ils relèvent les bilans avant-après favorables pour la sécurité en général (sans qu'on puisse isoler l'effet concernant les piétons) de la mise en place de systèmes lourds de type Bus Rapid Transit en position axiale sur de grandes infrastructures à Bogota (Colombie) et Guadalajara (Mexique). Ils notent cependant que l'effet d'un tel système est en grande partie imputable à la reconfiguration globale de ces infrastructures - mise en place d'un terre-plein central constituant un refuge pour la traversée des piétons, réduction des nombres de voies, forte diminution du nombre d'intersections, notamment. Notons aussi qu'à Bogota, selon ces auteurs, l'évaluation portait sur le passage d'un système de voies centrales réservées aux bus à un système de Bus Rapid Transit. Concernant Bogota, Bocarejo et al. relèvent une évolution de la répartition spatiale des accidents de piétons, avec l'apparition de nouvelles zones d'accumulation au niveau des stations du système de Bus Rapid Transit qui sont positionnées sur l'axe des avenues concernées [10].

Globalement, les résultats des travaux que nous venons de présenter, en particulier les plus solides d'entre eux (ceux de Devenport [5] et de Chen et al. [3]), suggèrent que l'implantation ou la présence de voies réservées aux bus dans le sens de la circulation générale tendent plutôt à dégrader la sécurité des piétons. Cette conclusion repose cependant sur peu d'études, menées pour partie dans des contextes urbains assez différents de ceux rencontrés dans les villes européennes.

\subsection{Voies bus à contresens de la circulation générale}

Concernant les voies réservées aux bus aménagées à contresens de la circulation générale, il fut très tôt question des problèmes que pouvait soulever cette configuration pour la sécurité des piétons [1] [11] [12]. Passalacqua évoque les premiers accidents graves de piétons survenus à Paris après l'implantation des premiers couloirs de bus à contresens à la fin des années 1960 [12]. Par exemple, le 11 octobre 1967 survient le premier accident mortel d'une piétonne dans le couloir de bus à contresens de la rue de Rivoli. Le couloir avait été installé le 2 octobre. Elle ne connaissait pas l'aménagement. La presse titre à l'époque : « Les couloirs de la mort ». D'autres accidents conduiront la municipalité à installer des barrières temporaires puis des chaînes le long de ce couloir à contre-courant. On compte à l'époque sur l'adaptation des piétons à cette innovation [1] [12].

Les premières publications scientifiques traitant des effets des couloirs de bus à contresens sur la sécurité des piétons datent des années 1980, et concernent des villes anglo-saxonnes. Les travaux menés par Berg et al. portent sur un seul couloir de bus à contresens, long de 0,9 mile, implanté en 1966 dans la ville de Madison (Wisconsin) sur une voie artérielle reliant les quartiers résidentiels de la ville au principal quartier d'affaires [11]. Les auteurs évaluent les effets sur la sécurité, non pas de la mise en place de cette voie bus, mais de sa fermeture au printemps 1979 pendant trois mois, suite à un fort sentiment d'insécurité des usagers. Les résultats suggèrent une réduction notable (mais non significative du fait de la période d'étude très brève) du nombre d'accidents corporels pour l'ensemble des usagers consécutivement à cette fermeture, par rapport à la même période de trois mois pour les quatre années précédentes ; mais les auteurs ne distinguent pas les accidents de piétons des autres accidents de la circulation. Toutefois, au cours des quatre années précédant la fermeture temporaire du couloir de bus à contresens, cinq bus étaient entrés en collision avec un piéton alors qu'ils circulaient dans ce couloir à contresens. En revanche, aucun accident entre piéton et bus n'avait été recensé au cours de la même période pour les bus circulant en sens inverse dans la voie de bus aménagée dans le sens de la circulation générale, ni pour les bus circulant sur une artère parallèle dépourvue de couloir de bus.

Les publications de LaPlante et Harrington [13] et de Kropidlowski [14] portent sur le centre-ville de Chicago où quatre couloirs de bus à contresens ont été mis en service en 1980 et 1981 dans un objectif d'amélioration de la qualité de l'air et de la vitesse commerciale des bus. Ces couloirs, longs de 1 à $1,5 \mathrm{~km}$, étaient séparés du reste de la circulation par un marquage au sol. Leur mise en service a été accompagnée d'un programme de formation des conducteurs de bus et d'une campagne d'information auprès de la population. Le nombre total d'accidents de

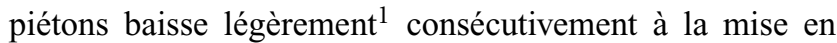
service des couloirs (après une hausse observée la première année), mais le nombre d'accidents de piétons heurtés par un bus augmente fortement, et se maintient à un niveau élevé [14]. Deux piétons sont d'ailleurs tués au cours de ces

\footnotetext{
${ }^{1}$ Cette tendance est à considérer avec précaution, l'évaluation n'ayant pas pris en compte la baisse générale des nombres d'accidents de piétons à Chicago à cette période.
} 
accidents. Le second, tué fin 1984, conduit la municipalité à supprimer les couloirs de bus à contresens et à les remplacer par des couloirs dans le sens de la circulation.

Une investigation rapportée par Brownfield et Devenport traite de dix rues équipées d'un couloir de bus à contresens à Londres, et suggère également un effet délétère de tels aménagements sur la sécurité des piétons [6]. Parmi les 276 accidents de piétons recensés au cours de trois années sur les dix sections étudiées, 114 (soit $41 \%$ ) se produisent du côté de la rue où le couloir est implanté. D'après les auteurs, compte tenu du très faible niveau de trafic dans la voie bus par rapport aux voies opposées, ce résultat suggère un risque élevé d'accidents de piétons dans le couloir de bus à contresens. D'autre part, dans ce couloir, la gravité des accidents (rapport entre le nombre de piétons tués ou gravement blessés et le nombre total de piétons blessés) est supérieure de $50 \%$ à celle observée dans les voies opposées. Cela s'explique notamment par le fait que, dans le couloir à contresens, la plupart des piétons blessés (71\%) ont été heurtés par des bus. Les auteurs observent aussi que dans $81 \%$ des accidents de piétons s'étant produits dans le couloir, le piéton est en début de traversée et descend du trottoir adjacent. Du côté opposé, cette proportion s'élève à $65 \%$. Cette différence s'explique d'après les auteurs par le caractère peu fréquenté de la voie bus et par la mise en service, à l'époque de l'étude, de bus équipés d'un moteur à l'arrière et donc plus silencieux en situation d'approche.

Plus récemment, concernant Paris et sa banlieue, Vayre met en évidence un risque de collision entre piétons et bus ${ }^{2}$ très supérieur (plus de six fois supérieur) dans les couloirs de bus à contresens par rapport aux couloirs dans le sens de la circulation ou aux voies générales; ce fort sur-risque est observé dans Paris intra-muros mais également en banlieue [7]. Enfin, à Mexico et à Porto-Alegre, les modélisations de Duduta et al. font également apparaître que la présence de couloirs de bus à contresens conduit à des taux d'accidents de piétons significativement supérieurs [9].

La conclusion qui se dégage de cet ensemble de résultats est que la présence d'un couloir de bus à contresens a une influence défavorable sur la sécurité des piétons. D'après les auteurs des différentes publications examinées, cet effet tiendrait à une mauvaise interprétation par les piétons du sens de circulation dans la voie bus, les conduisant à ne pas prendre d'information dans cette direction. C'est aussi ce que met en évidence le déroulement d'un cas d'accident décrit par Brenac et al., à relier au caractère peu lisible de la configuration des lieux avec quatre voies automobiles dans un sens et une voie bus à contresens [15]. Les observations de piétons conduites à Paris par Huguenin-Richard (2010) sur des sites complexes comportant des couloirs de bus

\footnotetext{
${ }^{2}$ Nombre de collisions entre piétons et bus rapporté au nombre de kilomètres parcourus par les bus.
}

à contresens, montrent également que dans ce genre de configuration, les conflits entre piétons et bus sont fréquents [16]. D'autre part, le fait que les voies bus restent des voies peu fréquentées par rapport aux voies automobiles à côté desquelles elles sont implantées et le caractère relativement silencieux des bus contemporains, équipés de moteurs situés à l'arrière, ne favorisent pas la détection des bus en approche par les piétons s'apprêtant à traverser. Finalement, les couloirs de bus à contresens semblent donc, près de 50 ans après la mise en service des premiers d'entre eux, toujours poser des problèmes de sécurité pour les piétons. L'adaptation des piétons à cet aménagement, sur laquelle on comptait à l'époque de leur lancement [1][12], ne semble pas s'être produite.

\subsection{Rues réservées aux bus}

Les études portant sur les effets de la mise en place de rues réservées aux transports collectifs (et ouvertes aux piétons, bien entendu) sur la sécurité sont rares et peu concluantes, et c'est encore plus vrai s'agissant des effets sur la sécurité des piétons. Il s'agit en général de rues traditionnelles fermées à la circulation automobile, les bus circulant sur la chaussée et les piétons se déplaçant plutôt sur les trottoirs. Une méta-analyse rapportée par Høye et al., portant sur un petit nombre d'observations américaines et scandinaves, ne met en évidence que des tendances non significatives (stagnation du nombre total d'accidents, baisse du nombre d'accidents de véhicules motorisés, augmentation du nombre d'accidents de piétons) [4]. Brownfield et Devenport rapportent des analyses concernant quatre rues commerçantes de Londres réservées aux bus [6]. La comparaison avec d'autres rues commerçantes ne porte que sur l'ensemble des accidents, impliquant tout type d'usager : la densité d'accidents par kilomètre de voies apparaît plus faible dans les rues réservées aux bus [6: 61]. Ces auteurs donnent d'autre part quelques informations sur les accidents de piétons dans ces rues réservées : les collisions entre bus et piétons représentent $60 \%$ des cas, et les chocs entre voitures et piétons environ un quart des cas (d'autres véhicules que les bus - riverains, véhicules de livraison - pouvant être autorisés dans ces rues). La gravité des blessures infligées aux piétons est particulièrement élevée dans les rues réservées (la proportion de piétons tués ou grièvement blessés, parmi l'ensemble des blessés, y est supérieure de $50 \%$, par rapport aux rues adjacentes). Cela est à relier notamment au type des véhicules impliqués, qui sont le plus souvent des bus.

$\mathrm{Du}$ fait de l'absence d'information sur les trafics et les mouvements de piétons, il est difficile de tirer des enseignements de ces différentes études concernant l'évolution des risques d'accidents de piétons résultant de 
l'aménagement de rues réservées aux bus et aux piétons. En effet, il est probable que la fermeture de rues au trafic automobile entraîne également, au-delà de la forte baisse du trafic motorisé, une augmentation de la fréquentation piétonne, comme cela a été observé pour les opérations de piétonisation [17].

\subsection{Conclusion de l'étude de la littérature antérieure}

Dans leur ensemble, les études présentées dans la présente section (section 2) suggèrent que les voies réservées aux bus, s'agissant du moins des couloirs de bus ordinaires et surtout des couloirs de bus à contresens, ont un effet plutôt défavorable sur la sécurité des piétons. Elles restent cependant assez peu nombreuses, portent en partie sur des infrastructures et des environnements urbains différents de ceux rencontrés en Europe, et ne contrôlent pas toujours les possibles facteurs de confusion (différences d'environnement urbain, différences ou variations des trafics et de la fréquentation piétonne). D'autre part, les déterminants des sur-risques observés dans ces études restent difficiles à cerner du fait de l'absence d'étude détaillée des accidents de piétons survenant sur ces types d'aménagement.

Nous proposons donc dans les sections suivantes une contribution sur ce sujet portant sur les aménagements réservés aux bus dans la ville de Marseille, comportant des analyses quantitatives des risques (contrôlant l'effet de l'environnement et des flux d'usagers), et une analyse qualitative des cas d'accidents concernés.

\section{Terrain d'étude, données et éléments généraux sur le réseau étudié}

Les investigations ont porté sur le réseau de la ville de Marseille où 27,3 $\mathrm{km}$ de voies réservées aux bus étaient en service en 2007. Plus précisément, les analyses réalisées concernent les rues de la ville de Marseille équipées entre 2007 et 2012 d'au moins un couloir de bus. Les $27,3 \mathrm{~km}$ de couloirs de bus se répartissent sur 20,5 km de rues, certaines rues pouvant comporter plusieurs couloirs. Trois principales configurations d'aménagement peuvent être identifiées dans le cas de Marseille (Fig. 1) :

- Les rues où les couloirs réservés sont aménagés dans le sens de la circulation, très généralement à droite des autres voies de circulation. Nous parlerons dans la suite du texte de «couloirs de bus ordinaires ». Cette configuration représente près de $23 \mathrm{~km}$ de couloirs de bus, sur $17,6 \mathrm{~km}$ de rues ;

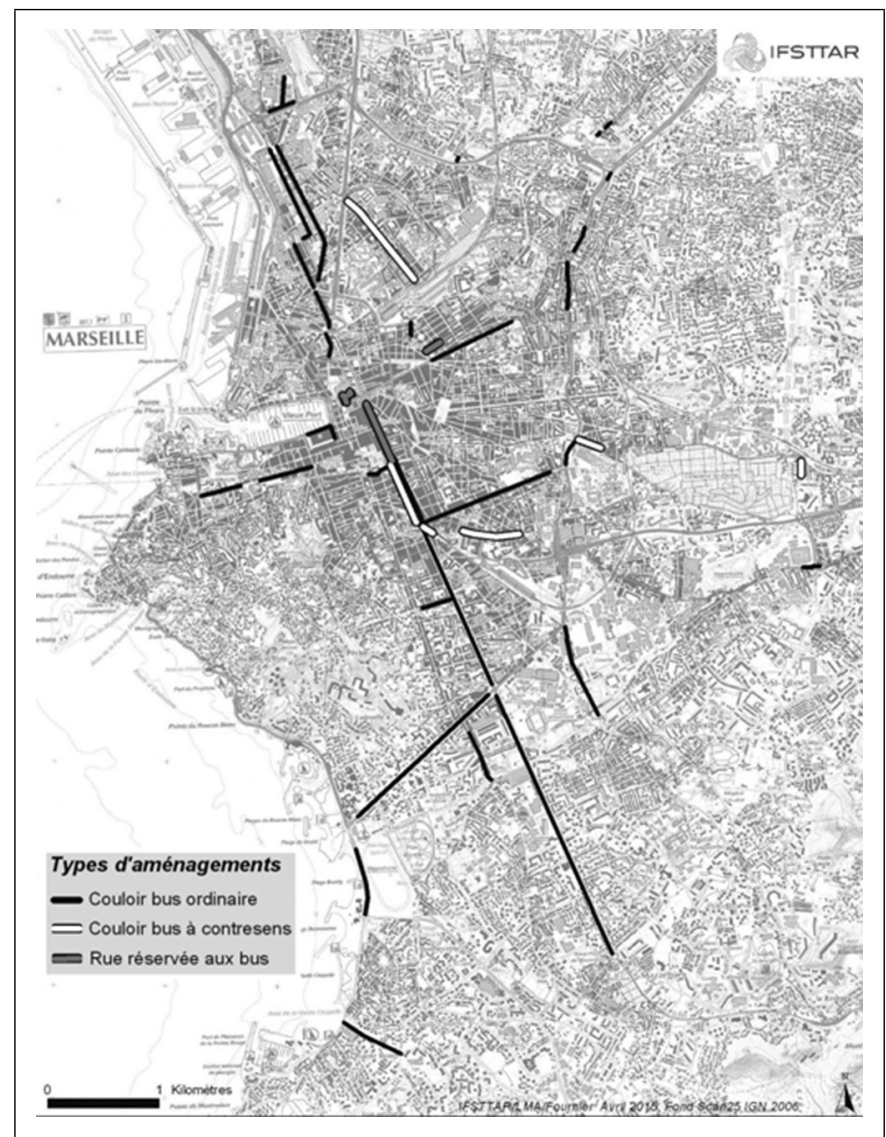

Fig. 1 Rues de Marseille équipées en 2007 de voies réservées aux bus

Nota : Deux tronçons équipés de couloirs de bus ordinaires, situés hors des limites de cette carte, n'ont pas pu être représentés.

- Les rues où les couloirs réservés sont aménagés à contresens de la circulation générale. Cette configuration représente environ $2,5 \mathrm{~km}$ de couloirs de bus, sur 2,5 $\mathrm{km}$ de rues (dont 0,6 km comportent aussi un couloir de bus ordinaire en vis-à-vis et sont aussi comptés dans les $17,6 \mathrm{~km}$ de rues de la catégorie précédente);

- Les rues où les couloirs réservés sont les seules voies de circulation. Nous parlerons dans la suite du document de « rues réservées aux bus ». Cette configuration représente plus de $1,8 \mathrm{~km}$ de couloirs de bus, correspondant à $1 \mathrm{~km}$ de rues, principalement situées dans l'hypercentre de la ville.

Outre les bus, les taxis et véhicules d'urgence sont aussi autorisés à circuler dans ces voies réservées. Sur quelques sections, la circulation des vélos y est parfois également autorisée.

Deux principales catégories de données ont été collectées dans cette recherche : des données d'accidents et des données de trafic routier. Pour les données d'accidents, 
nous avons eu recours aux procédures d'accidents corporels (procès-verbaux) établies par les forces de l'ordre au cours des années 2007 à 2012. L'ensemble des cas impliquant un piéton et se déroulant dans une rue équipée d'un couloir de bus - qu'ils surviennent dans le couloir ou sur les autres files - ont été sélectionnés et localisés dans un système d'information géographique. L'analyse des données d'accidents a porté sur les jours de semaine (du lundi au samedi, hors jours fériés) et sur les heures de journée (entre $6 \mathrm{~h}$ et $22 \mathrm{~h}$ ) : seuls les accidents survenus durant ces périodes ont été pris en compte.

Les données de trafic routier sont issues des boucles de comptage implantées sur le réseau routier principal de la ville. Les véhicules circulant dans le couloir de bus n'étant pas comptabilisés par ces boucles (elles sont placées sur les voies de circulation générale), il a été nécessaire de procéder à des comptages manuels complémentaires pour pouvoir réaliser les analyses rapportées dans les sections suivantes (sections 4 et 5). Nous avons donc conduit une campagne d'observations afin d'estimer la part que représente le trafic de la voie réservée aux bus dans le trafic total. Ces comptages ont été réalisés durant l'année $2011^{3}$, sur neuf axes, avec un plan d'observations visant à rendre compte des trafics des jours ouvrables (du lundi au samedi, hors jours fériés) entre $6 \mathrm{~h}$ et $22 \mathrm{~h}$. Des informations plus détaillées sur la méthode d'observation et sur les résultats sont rapportées dans la référence [18].

Avant de passer aux analyses plus formelles traitées dans les sections suivantes, quelques éléments descriptifs généraux peuvent être présentés ici concernant les rues de la ville de Marseille équipées de couloirs de bus. Le tableau 1 donne, par type de couloir ou voie réservée, les longueurs de rue concernées, les nombres d'accidents de piéton correspondants sur la période 2007-2012, et la densité moyenne d'accidents de piéton par kilomètre de voirie.

Ce tableau montre que, rapporté à la longueur des tronçons étudiés, le nombre moyen annuel d'accidents de piétons s'élève à environ $2,8 \mathrm{acc} / \mathrm{an} / \mathrm{km}$ pour les tronçons de rues équipées de couloirs de bus ordinaires, 3,7 acc/an $/ \mathrm{km}$ pour les tronçons équipés de couloirs de bus à contresens, et $5,2 \mathrm{acc} / \mathrm{an} / \mathrm{km}$ pour les rues réservées. Cependant, il faut noter que ces différences sont sans doute en partie le reflet d'une différence de centralité entre les diverses configurations d'aménagement : en effet, certains couloirs de bus ordinaires sont situés en dehors de l'hypercentre de la ville, contrairement aux deux autres types de configurations que l'on rencontre exclusivement dans les secteurs centraux,

\footnotetext{
${ }^{3} \mathrm{~L}$ 'hypothèse a été faite que la part du trafic comptabilisé dans la voie réservée était similaire entre l'année 2011 au cours de laquelle les observations ont été réalisées et les autres années de la période d'étude (2007 à 2012).
}

dans des environnements très commerçants où les piétons sont plus nombreux (Fig. 1).

Dans les rues équipées de couloirs de bus à contresens, on peut relever d'autre part que près de la moitié (environ $49 \%$ ) des accidents de piétons recensés sur ces tronçons se produisent dans le couloir à contresens ${ }^{4}$, bien que le trafic dans ce couloir réservé (ouvert aux seuls bus, taxis et véhicules d'urgence) soit faible par rapport au trafic automobile sur les voies de sens opposé. Cela suggère l'existence d'un risque élevé de collision contre un piéton pour les véhicules circulant dans le couloir de bus à contresens.

Mais, dans l'ensemble, les éléments descriptifs présentés dans cette section ne peuvent pas être interprétés en termes de niveau de sécurité piétonne des rues équipées de voies réservées aux bus, faute de pouvoir rapporter les densités d'accidents aux volumes de trafic ${ }^{5}$ et au niveau de fréquentation piétonne. Dans les sections suivantes, nous tentons de faire émerger des résultats plus informatifs, en prenant en considération les trafics, et en contrôlant l'effet des environnements urbains et des fréquentations piétonnes correspondantes.

\section{Risques d'accident de piéton sur les sections équipées de couloirs de bus ordinaires}

Nous cherchons ici à évaluer les risques d'accidents de piéton sur des sections de voirie équipées de couloirs de bus ordinaires, par rapport à des sections témoins non équipées (ne comportant que des voies banalisées), pour un même environnement urbain et une même fréquentation piétonne. Cette analyse n'a pas été étendue aux autres configurations de voies réservées, car il était très difficile de trouver des sections témoins appropriées pour les sections comportant un couloir de bus à contresens et pour les sections de rues réservées aux bus.

\subsection{Aspects méthodologiques et données}

Les analyses portent sur des sections en vis-à-vis, c'est-àdire situées sur un même axe et l'une en face de l'autre, l'une étant équipée d'un couloir réservé aux bus aménagé à droite des voies banalisées, l'autre, de sens inverse et composée uniquement de voies banalisées, constituant la section témoin. L'intérêt de cette méthode est qu'elle permet de mieux contrôler l'effet du contexte urbain sur le taux d'accident, dans le sens où les sections équipées

\footnotetext{
${ }^{4}$ Dans les rues équipées de couloirs de bus ordinaires, la proportion d'accidents de piétons survenus dans ces couloirs est de $24 \%$.

${ }^{5}$ Nous ne disposions pas de données de trafic sur l'ensemble de ces infrastructures.
} 
Tableau 1 Longueur des rues équipées de voies réservées aux bus, effectifs d'accidents de piétons (2007-2012), et densité d'accidents de piétons pour chaque configuration

\begin{tabular}{lccr}
\hline Types d'aménagement & $\begin{array}{l}\text { Longueur } \\
(\mathbf{k m})\end{array}$ & $\begin{array}{l}\text { Nombre d'accidents } \\
\text { de piéton }\end{array}$ & $\begin{array}{l}\text { Densité d'accidents (acc/an/km) } \\
\text { (et intervalle de confiance à 95 \%) }\end{array}$ \\
\hline $\begin{array}{l}\text { Couloirs de bus } \\
\text { ordinaires }\end{array}$ & 17,6 & 263 & $2,84(2,51 ; 3,21)$ \\
$\begin{array}{l}\text { Couloirs de bus à } \\
\text { contresens }\end{array}$ & 2,5 & 49 & $3,68(2,72 ; 4,86)$ \\
\begin{tabular}{l} 
Rues réservées aux bus \\
\hline
\end{tabular} & 1,0 & 28 & $5,19(3,45 ; 7,51)$ \\
\hline
\end{tabular}

Nota : Du fait de travaux ou de transformations, certaines années ont été écartées pour certaines rues. Le nombre moyen d'années d'observation n'est donc pas de 6 ans, mais de 5,26 ans pour les couloirs de bus ordinaires, de 5,38 ans pour les couloirs de bus à contresens, et de 5,44 ans pour les rues réservées aux bus.

de couloirs et les sections témoins se situent ainsi dans le même environnement. D'autre part, elle présente également l'avantage de comparer des sections sur lesquelles les traversées de piétons sont, en nombre, équivalentes, puisque chaque piéton traversant la section équipée d'un couloir traverse également la section témoin.

Les critères de sélection des axes étaient les suivants :

- circulation sur l'axe à double sens ;

- aménagement de l'espace viaire avec au moins deux voies de circulation par sens ;

- présence dans un sens d'une section équipée d'un couloir de bus aménagé à droite des voies de circulation et dans l'autre sens (en face) d'une section comportant uniquement des voies banalisées (section témoin);

- longueur des sections au moins égale à 200 mètres ;

- comparabilité de l'environnement des voies entre les deux sens ;

- disponibilité de données d'accidents et de trafic.

Ces critères nous ont conduits à la sélection de 16 sections parmi l'ensemble des sections candidates, soit huit sections équipées d'un couloir de bus ordinaire (représentant 4650 mètres de couloirs réservés) et huit sections témoins. Le tableau 2 présente les caractéristiques de ces sections.

Deux types de risques ont été évalués :

(a) Le risque relatif ${ }^{6}$ pour un véhicule, par kilomètre parcouru, de heurter et de blesser un piéton (ou plusieurs) sur une section équipée, par rapport à la section témoin. Pour cela, nous avons estimé le taux d'accidents de piétons (rapport entre le nombre de collisions contre

6 Pour des raisons de simplicité et d'accessibilité, nous utilisons ici le terme de risque relatif, dans un sens informel; si l'on s'en tient à la terminologie usuelle en épidémiologie, il s'agit plutôt d'un taux d'incidence relatif (incidence rate ratio). des piétons recensées au cours des années 2007 à 2012, en jour ouvrable entre $6 \mathrm{~h}$ et $22 \mathrm{~h}$, et le nombre de véhicules-kilomètres sur ces sections au cours des mêmes périodes). Ce taux, calculé sur la section équipée de couloir de bus $\left(t_{\mathrm{scb}}\right)$ puis sur la section témoin $\left(t_{\mathrm{stem}}\right)$, permet ensuite de calculer le risque relatif (taux relatif) sur la section équipée par rapport à la section témoin : $t_{\text {scb }} / t_{\text {stem }}$. Une valeur de 1,10 , par exemple, signifierait que le risque pour un véhicule de heurter un piéton est supérieur de $10 \%$ sur la section équipée par rapport à la section témoin. Compte tenu des effectifs d'accidents relativement faibles, les résultats étant rarement significatifs pour chaque paire de sections prise individuellement, nous avons également estimé un risque relatif commun pour l'ensemble des paires de sections, en appliquant les techniques usuellement employées pour l'agrégation de résultats lors de méta-analyses (agrégation de rapports de taux ; voir notamment [19][20][21] $)$. Nous avons également examiné l'éventuelle hétérogénéité statistique des résultats entre les paires de sections au moyen de la statistique $Q$ [22].

(b) Le risque relatif, pour un piéton traversant, d'être heurté (et blessé) sur la section équipée, par rapport à la section témoin. Ce risque peut être calculé puisque le nombre de piétons traversant est le même sur les deux sections en vis-à-vis. Si $n_{\mathrm{scb}}$ représente le nombre de piétons traversant heurtés sur la section équipée, et $n_{\text {stem }}$ le nombre de piétons traversant heurtés sur la section témoin, ce risque relatif peut être estimé par le rapport $n_{\text {scb }} / n_{\text {stem }}$. De la même façon, nous avons calculé ces risques relatifs pour chaque paire de sections, puis un risque relatif commun (en examinant aussi l'éventualité

7 Concernant la méthode utilisée pour l'agrégation, nous avons utilisé l'estimateur de Mantel-Haenszel, qui paraît préférable dans le cas d'effectifs limités [20]. 
d'une hétérogénéité entre les paires de sections) avec les mêmes techniques de méta-analyse.

\subsection{Résultats}

Le tableau 3 présente le nombre d'accidents constatés et la circulation (en milliers de véhicules-kilomètres) pour les sections équipées de couloirs de bus ordinaires et pour les sections témoins correspondantes, ainsi que les résultats obtenus concernant les risques de collision avec un piéton. Au total, 51 accidents impliquant un piéton se sont produits entre 2007 et 2012 sur les huit sections équipées d'un couloir de bus, et 32 accidents sont survenus sur les huit sections témoins correspondantes. Le nombre élevé d'accidents sur la paire de sections $n^{\circ} 8$ s'explique en partie par la longueur de ces sections, et en partie par le fait qu'elles sont situées dans un secteur central de la ville (les accidents de piétons étant généralement plus nombreux dans les zones urbaines les plus denses).

Le risque relatif, pour un véhicule, de heurter un piéton sur la section équipée d'un couloir de bus ordinaire, par rapport à la section témoin correspondante (non équipée), apparaît supérieur à un dans cinq cas sur huit, suggérant l'existence d'un sur-risque pour les sections équipées ; les intervalles de confiance de ces risques relatifs sont cependant très larges. La méta-analyse appliquée à l'ensemble des sections donne un risque relatif commun de 1,67 (intervalle de confiance à $95 \%: 1,08$ à 2,58 ). Le test d'hétérogénéité suggère de ne pas rejeter l'hypothèse nulle d'homogénéité du risque relatif entre les différentes paires de sections $(Q=10,23 ; p=0,18)$. Cet ensemble de résultats tend à montrer que le risque, pour un véhicule, de heurter un piéton est plus élevé sur une section équipée d'un couloir de bus que sur une section similaire non équipée, du moins entre $6 \mathrm{~h}$ et $22 \mathrm{~h}$ les jours ouvrables (du lundi au samedi, hors jours fériés).

Les estimations du risque relatif, pour un piéton traversant, d'être heurté (et blessé) par un véhicule sur les sections équipées d'un couloir bus ordinaire, par rapport aux sections témoins appariées, non équipées, ont été également calculées. Seuls les piétons blessés lors de traversée de chaussée ont été pris en compte dans ces calculs. Ces risques relatifs sont supérieurs à 1 pour 5 des 8 paires de sections considérées ; les intervalles de confiance sont larges et contiennent tous la valeur 1 , et on ne peut donc conclure pour chaque paire de sections considérée individuellement. L'agrégation des différentes paires de sections conduit ici à un risque relatif commun de 1,42 (avec un intervalle de confiance de 0,90 à 2,25), non significativement différent de 1 . Le test d'hétérogénéité suggère de ne pas rejeter l'hypothèse nulle d'homogénéité du risque relatif entre les différentes paires de sections $(Q=5,74 ; p=0,57)$. Ces derniers résultats ne permettent pas de conclure de façon certaine qu'un piéton traversant une section équipée d'un couloir de bus ordinaire coure un risque de se faire heurter et blesser supérieur à celui qu'il encourt en traversant une section comparable non équipée.

Dans leur ensemble, les résultats présentés dans cette section paraissent cependant en relative cohérence avec les études antérieures, qui suggèrent que l'aménagement de couloirs de bus dans le sens de la circulation générale tend à dégrader la sécurité des piétons (voir notamment les solides études avant-après de Devenport et de Chen et al. [5] [3]). L'étude détaillée des accidents de piétons présentée dans la section 6 fournit quelques éléments d'interprétation des sur-risques mis en évidence pour les sections équipées de couloirs de bus ordinaires.

\section{Risques d'accidents de piéton liés à la circulation dans les couloirs de bus}

Les politiques de mobilité durable tendent à encourager de nouvelles formes de déplacements (covoiturage, autopartage, véhicules électriques en libre-service, etc.). Cette évolution donne une certaine actualité à la question de savoir si les couloirs de bus doivent être ouverts, au-delà de leurs principaux destinataires (bus, taxis), à d'autres circulations, comme celle des véhicules électriques ou celle des véhicules à fort taux d'occupation, par exemple. Une telle extension de l'usage des couloirs de bus pourrait-elle être dommageable à la sécurité des piétons ?

Cette section vise à apporter une contribution sur ce point, en tentant de répondre à une question spécifique : la circulation d'un véhicule dans un couloir de bus engendre-t-elle un risque supérieur d'accident de piéton, par rapport à la circulation d'un véhicule dans une voie banalisée adjacente ? Plus précisément, nous tentons d'estimer le risque pour un véhicule, par kilomètre parcouru dans un couloir de bus, de heurter et de blesser un piéton (ou plusieurs), par rapport au risque par kilomètre parcouru dans les voies banalisées adjacentes de même sens ${ }^{8}$.

Le risque relatif ${ }^{9}$ a été estimé selon les méthodes déjà décrites dans la section précédente (sous-section 4.1), les voies banalisées de même sens adjacentes au couloir de

\footnotetext{
${ }^{8} \mathrm{~L}$ 'analyse se concentre sur les couloirs de bus ordinaires, implantés dans le sens de la circulation générale. Nous ne disposions pas de données de trafic suffisantes pour procéder à une telle analyse pour les couloirs à contresens. Cependant, l'existence d'un sur-risque lié à la circulation dans les couloirs de bus à contresens ressort assez clairement du résultat général exposé dans la section 3 (le fait que, dans les rues équipées de couloirs de bus à contresens, $49 \%$ des accidents de piétons se produisent dans le couloir de bus à contresens lui-même, plutôt que dans les voies banalisées de sens opposé).

${ }^{9}$ Ici aussi, pour des raisons de simplicité et d'accessibilité, nous employons les termes de risque et de risque relatif, alors qu'il s'agit, stricto sensu, de taux d'incidence et de taux d'incidence relatif.
} 
Tableau 2 Présentation des huit sections équipées de voies réservées aux bus et des huit sections témoins correspondantes

\begin{tabular}{|c|c|c|c|c|}
\hline \multirow[t]{2}{*}{ Paire de sections } & \multicolumn{2}{|c|}{$\begin{array}{l}\text { Section équipée d'un couloir de bus } \\
\text { ordinaire }\end{array}$} & \multicolumn{2}{|l|}{ Section témoin } \\
\hline & Nombre de voies & Longueur (m) & Nombre de voies & Longueur (m) \\
\hline $\mathrm{N}^{\mathrm{o}} 1$ & 2 voies plus 1 voie bus & 300 & 3 voies & 300 \\
\hline $\mathrm{N}^{\mathrm{O}} 2$ & 2 voies plus 1 voie bus & 800 & 3 voies & 800 \\
\hline $\mathrm{N}^{\mathrm{O}} 3$ & 2 voies plus 1 voie bus & 400 & 3 voies & 400 \\
\hline $\mathrm{N}^{\mathrm{O}} 4$ & 1 voie plus 1 voie bus & 450 & 2 voies & 450 \\
\hline $\mathrm{N}^{\mathrm{O}} 5$ & 2 voies plus 1 voie bus & 400 & 3 voies & 400 \\
\hline $\mathrm{N}^{\mathrm{O}} 6$ & 2 voies plus 1 voie bus & 250 & 3 voies & 250 \\
\hline $\mathrm{N}^{\mathrm{o}} 7$ & 2 voies plus 1 voie bus & 600 & 3 voies & 600 \\
\hline $\mathrm{N}^{\mathrm{O}} 8$ & 2 voies plus 1 voie bus & 1100 & 2 voies & 1100 \\
\hline
\end{tabular}

Tableau 3 Estimation du risque relatif, pour un véhicule, de collision avec un piéton sur les sections équipées d'un couloir de bus ordinaire par rapport aux sections témoins correspondantes (les données portent sur la période 2007-2012, les jours ouvrables, entre $6 \mathrm{~h}$ et $22 \mathrm{~h}$ )

Section équipée d'un couloir de bus ordinaire Section témoin

Risque relatif de heurter et blesser un piéton(et $i$.

c. à $95 \%$ )

\begin{tabular}{|c|c|c|c|c|c|}
\hline $\begin{array}{l}\text { Paire de } \\
\text { sections }\end{array}$ & Accidents de piéton & $\begin{array}{l}\text { Circulation (en milliers } \\
\text { de véhicules-kilomètres) }\end{array}$ & $\begin{array}{l}\text { Accidents } \\
\text { de piéton }\end{array}$ & $\begin{array}{l}\text { Circulation (en milliers } \\
\text { de véhicules-kilomètres) }\end{array}$ & \\
\hline $\mathrm{N}^{\mathrm{O}} 1$ & 3 & 8234 & 0 & 6789 & $-*$ \\
\hline $\mathrm{N}^{\mathrm{O}} 2$ & 5 & 18421 & 11 & 17266 & $0,43(0,15 ; 1,23)$ \\
\hline $\mathrm{N}^{\mathrm{O}} 3$ & 2 & 13071 & 2 & 10403 & $0,80(0,11 ; 5,65)$ \\
\hline $\mathrm{N}^{\mathrm{O}} 4$ & 5 & 9527 & 1 & 8904 & $4,67(0,55 ; 40,00)$ \\
\hline $\mathrm{N}^{\mathrm{o}} 5$ & 9 & 24793 & 5 & 34517 & $2,51(0,84 ; 7,48)$ \\
\hline $\mathrm{N}^{\mathrm{O}} 6$ & 5 & 6503 & 2 & 9678 & $3,72(0,72 ; 19,18)$ \\
\hline $\mathrm{N}^{\mathrm{O}} 7$ & 4 & 25865 & 2 & 31201 & $2,41(0,44 ; 13,18)$ \\
\hline \multirow[t]{2}{*}{$\mathrm{N}^{\mathrm{o}} 8$} & 18 & 27384 & 9 & 25829 & $1,89(0,85 ; 4,20)$ \\
\hline & & & & Risque relatif commun : & $1,67(1,08 ; 2,58)$ \\
\hline
\end{tabular}

*Ne peut être estimé (division par zéro).

bus étant utilisées comme témoins. L'analyse a porté sur 13 sections de rues équipées de couloirs de bus dans le sens de la circulation générale (Tableau 4). Par section, nous entendons l'ensemble constitué par le couloir de bus et les voies adjacentes de même sens, généralement au nombre de deux. L'échantillon est plus large que dans la partie précédente (partie 4), car il n'était pas nécessaire ici de disposer d'une section non équipée en vis-à-vis de chacune des sections considérées ${ }^{10}$.

${ }^{10}$ Mais le linéaire de sections équipées étudiées dans la partie 4 est inclus dans les 13 sections étudiées ici.
Au cours des années 2007 à 2012, 122 accidents de piétons ont été recensés sur les 13 sections étudiées. Parmi eux, 35 cas, soit $29 \%$, impliquent un véhicule circulant dans le couloir de bus et heurtant un piéton. Le tableau 5 présente les effectifs d'accidents de piétons recensés sur chacune des sections étudiées et les niveaux de trafics dans les couloirs de bus et dans les voies banalisées. L'estimation des risques relatifs sur chacune des sections est également présentée.

La méta-analyse effectuée sur l'ensemble des sections étudiées donne un risque relatif global de 3,44 avec un intervalle de confiance (IC) à $95 \%$ de 2,30 à 5,15. Le test d'hétérogénéité réalisé ne permet pas de conclure dans le 


\begin{tabular}{|c|c|c|}
\hline Section & Nombre de voies & Longueur (m) \\
\hline A & 2 voies plus 1 voie réservée aux bus & 1500 \\
\hline B & 2 voies plus 1 voie réservée aux bus & 1500 \\
\hline $\mathrm{C}$ & 2 voies plus 1 voie réservée aux bus & 800 \\
\hline $\mathrm{D}$ & 2 voies plus 1 voie réservée aux bus & 1300 \\
\hline $\mathrm{E}$ & 2 voies plus 1 voie réservée aux bus & 2500 \\
\hline $\mathrm{F}$ & 2 voies plus 1 voie réservée aux bus & 1900 \\
\hline G & 1 voie plus 1 voie réservée aux bus & 450 \\
\hline $\mathrm{H}$ & 2 voies plus 1 voie réservée aux bus & 650 \\
\hline I & 2 voies plus 1 voie réservée aux bus & 850 \\
\hline $\mathrm{J}$ & 2 voies plus 1 voie réservée aux bus & 1200 \\
\hline $\mathrm{K}$ & 1 voie plus 1 voie réservée aux bus & 450 \\
\hline $\mathrm{L}$ & 1 voie plus 1 voie réservée aux bus & 350 \\
\hline M & 1 voie plus 1 voie réservée aux bus & 600 \\
\hline
\end{tabular}

sens d'une hétérogénéité du résultat selon les différentes sections $(Q=13,86$ et $p=0,31)$. Cela suggère que la tendance observée peut être considérée comme commune à l'ensemble des sections. Les véhicules circulant dans les couloirs de bus implantés dans le sens de la circulation ont donc, durant les jours de semaine et au cours des heures de journée, près de 3,5 fois plus de chance de heurter un piéton que les véhicules circulant dans les voies banalisées adjacentes, sur ce terrain d'études du moins.

On peut répéter le même type d'analyse pour diverses catégories de véhicules, pour l'estimation de leur risque relatif de collision avec un piéton lors de la circulation dans les couloirs de bus. Cela conduit cependant à subdiviser un échantillon global d'accidents déjà relativement limité, ce qui entraîne une perte de puissance statistique. Si l'on considère les véhicules de tourisme ou utilitaires non autorisés à circuler dans ces couloirs, le risque relatif de collision avec un piéton est de 10,08 (IC à $95 \%: 4,99$ à 20,33). Quant au risque relatif pour les véhicules autorisés à circuler dans les couloirs de bus, il s'élève sur l'ensemble des sections étudiées à 2,56 (IC à $95 \%: 0,66$ à 9,86); au sein de cette catégorie, les risques relatifs obtenus pour les bus et les taxis sont respectivement de 2,72 $(0,24$ à 30,72$)$ et $1,63(0,40$ à 6,72$)$, et celui concernant les ambulances est de $0,73(0,05$ à 11,60$)$. Dans cet ensemble de résultats, les tests d'hétérogénéité ne conduisent pas à remettre en cause l'hypothèse d'homogénéité du risque relatif sur les différentes sections. On voit que tous ces intervalles de confiance se recouvrent largement, et il est difficile de conclure

\begin{tabular}{|c|c|c|c|c|c|}
\hline \multirow[b]{2}{*}{ Section } & \multicolumn{2}{|c|}{ Couloir de bus } & \multicolumn{2}{|c|}{ Voies banalisées adjacentes } & \multirow{2}{*}{$\begin{array}{l}\text { Risque relatif de heurter et } \\
\text { blesser un piéton (et i. c. à } 95 \% \text { ) }\end{array}$} \\
\hline & $\begin{array}{l}\text { Accidents de } \\
\text { piéton }\end{array}$ & $\begin{array}{l}\text { Circulation (en milliers } \\
\text { de véhicules-kilomètres) }\end{array}$ & $\begin{array}{l}\text { Accidents de } \\
\text { piéton }\end{array}$ & $\begin{array}{l}\text { Circulation (en } \\
\text { milliersde } \\
\text { véhicules-kilomètres) }\end{array}$ & \\
\hline A & 3 & 2863 & 16 & 34861 & $2,28(0,67 ; 7,83)$ \\
\hline B & 5 & 2457 & 22 & 34130 & $3,16(1,20 ; 8,34)$ \\
\hline $\mathrm{C}$ & 1 & 296 & 2 & 12087 & $20,43(1,85 ; 225,31)$ \\
\hline $\mathrm{D}$ & 0 & 1270 & 6 & 20414 & $0,00-*$ \\
\hline $\mathrm{E}$ & 1 & 4905 & 11 & 70139 & $1,30(0,17 ; 10,07)$ \\
\hline $\mathrm{F}$ & 1 & 3796 & 1 & 40732 & $10,73(0,67 ; 171,57)$ \\
\hline $\mathrm{G}$ & 1 & 720 & 3 & 7700 & $3,56(0,37 ; 34,24)$ \\
\hline $\mathrm{H}$ & 4 & 2825 & 6 & 17287 & $4,08 \quad(1,15 ; 14,46)$ \\
\hline I & 1 & 2482 & 0 & 26244 & $-*{ }^{*} *$ \\
\hline $\mathrm{J}$ & 8 & 2627 & 8 & 24586 & $9,36(3,51 ; 24,94)$ \\
\hline K & 2 & 639 & 1 & 6094 & $19,08(1,73 ; 210,42)$ \\
\hline $\mathrm{L}$ & 1 & 678 & 3 & 5464 & $2,68(0,28 ; 25,81)$ \\
\hline \multirow[t]{2}{*}{ M } & 7 & 4693 & 8 & 9841 & $1,83(0,67 ; 5,06)$ \\
\hline & & & & Risque relatif commun : & $3,44 \quad(2,30 ; 5,15)$ \\
\hline
\end{tabular}


de façon ferme à l'existence de différences de risque relatif entre ces catégories de véhicules, même si ces résultats tendent à suggérer que les véhicules de tourisme ou utilitaires non autorisés ont probablement un risque relatif supérieur.

Pour les deux-roues motorisés, le risque relatif de collision avec un piéton lié à la circulation dans un couloir de bus est de 2,26 (IC à $95 \%: 1,15$ à 4,46). Le test d'hétérogénéité ne conduit pas non plus à remettre en cause l'hypothèse d'homogénéité de ce risque relatif sur les différentes sections.

Les résultats présentés dans cette section suggèrent donc que la circulation d'un véhicule dans un couloir de bus est nettement plus défavorable à la sécurité des piétons que la circulation d'un véhicule dans une voie banalisée adjacente. Cette conclusion est à tempérer dans la mesure où le risque élevé dans le couloir de bus pourrait tenir en partie au fait que les usagers y circulant sont pour une forte part des usagers non autorisés ${ }^{11}$, dont le comportement général pourrait être supposé moins sûr que celui des usagers circulant dans les voies banalisées. L'étude détaillée des cas d'accidents de piétons, présentée au point suivant, suggère cependant que le risque élevé de collision avec un piéton dans les couloirs de bus tient aussi à des aspects structurels, et en particulier aux vitesses plus élevées dans les couloirs de bus par rapport aux voies adjacentes, plus souvent congestionnées.

Bien entendu, les effets de l'ouverture des couloirs de bus à certaines catégories de véhicules sur la sécurité des piétons ne peuvent être directement inférés des résultats présentés dans cette section, sans prendre en compte l'influence éventuelle d'une telle ouverture sur l'ensemble des circulations (par exemple, la circulation d'un véhicule à fort taux d'occupation dans un couloir de bus pourrait, en principe, se substituer à la circulation de plusieurs véhicules dans les voies banalisées adjacentes).

\section{Processus d'accidents de piéton dans les voies réservées aux bus}

Afin de mieux cerner les déterminants des risques mis en évidence par les analyses précédentes et de mieux comprendre la contribution des couloirs de bus et de leur aménagement dans le déroulement des accidents de piétons, une analyse approfondie des cas d'accidents se produisant dans les voies réservées aux bus a été entreprise. Cette analyse qualitative s'est appuyée sur les rapports de police (procès-verbaux d'accident), comportant notamment des données textuelles (résumé du cas par les forces de l'ordre,

\footnotetext{
${ }^{11}$ Parmi les cas du tableau 5, les usagers non autorisés (y compris les deux-roues motorisés) représentent environ les deux tiers des impliqués dans des collisions avec des piétons dans le couloir de bus.
}

procès-verbaux d'audition des impliqués et témoins) et des schémas, l'ensemble permettant généralement d'accéder à une bonne compréhension du déroulement de chaque cas. Contrairement aux analyses précédentes, les investigations ont porté uniquement sur trois années, l'étude approfondie de cas d'accident étant très coûteuse en temps.

Toutes les configurations d'aménagement des couloirs ont été examinées (couloirs dans le sens de la circulation, couloirs de bus à contresens et rues réservées). Chaque cas d'accident impliquant un piéton et se produisant dans une voie réservée a fait l'objet d'une analyse approfondie selon un modèle d'analyse en phases [23]. Les cas présentant un même air de famille et jugés similaires dans leur déroulement ont ensuite été regroupés et ont donné lieu à la description de scénarios types d'accidents. Nous ne reviendrons pas ici sur la méthode employée pour le regroupement des cas jugés similaires et la description de scénarios types d'accidents. Pour davantage de détails sur cet aspect, le lecteur pourra se reporter aux références [24] et [15]. Une cartographie des accidents selon leur scénario type a également été conduite afin d'apporter des éclairages sur les liens entre processus d'accidents, configurations d'aménagement des couloirs de bus et situation dans la ville.

Parmi les 209 accidents de piétons s'étant produits au cours des trois années étudiées dans les rues de Marseille équipées de voies réservées aux bus, 71 cas se sont produits dans une voie réservée ( 39 dans les couloirs de bus ordinaires, 13 dans les couloirs de bus à contresens et 19 dans les rues réservées aux bus). Parmi ces 71 cas, 27 impliquent un deux-roues motorisé, 19 impliquent un bus, 15 impliquent une voiture particulière, 4 impliquent un taxi, 2 impliquent un vélo, 3 impliquent des véhicules d'urgence. Dans un cas, le type de véhicule heurtant le piéton n'a pu être déterminé. Dans environ $60 \%$ des cas, le véhicule heurtant le piéton n'était donc pas autorisé à circuler dans la voie réservée.

L'analyse de chacun des cas et le regroupement des cas similaires en scénarios types montrent que neuf scénarios types d'accidents peuvent être distingués, dans les voies réservées aux bus. Trois des neuf scénarios types représentent $61 \%$ des cas (Tableau 6). Ces scénarios types, notés PCB1, PCB2 et PCB3, représentent respectivement $30 \%, 20 \%$ et $11 \%$ des cas.

Le scénario type PCB1 correspond à des accidents entre un piéton traversant un couloir de bus à contresens ou une rue réservée aux bus sans détecter un véhicule qui y circule. La description détaillée de ce scénario type est présentée dans le tableau 7.

Le scénario type PCB2 correspond à des accidents entre un piéton traversant dans une circulation dense et un véhicule circulant dans un couloir de bus aménagé dans le sens général. Le piéton et le conducteur du véhicule se perçoivent souvent très tardivement en raison des files de véhicules arrêtés ou fortement ralentis sur les voies 
Tableau 6 Répartition dans les différents scénarios types des accidents survenus dans les voies réservées aux bus

\begin{tabular}{lrr}
\hline Scénario type & Effectif & Pourcentage \\
\hline PCB1 & 17 & $(30,4 \%)$ \\
PCB2 & 11 & $(19,6 \%)$ \\
PCB3 & 6 & $(10,7 \%)$ \\
PCB4 & 5 & $(8,9 \%)$ \\
PCB5 & 5 & $(8,9 \%)$ \\
PCB6 & 2 & $(3,6 \%)$ \\
PCB7 & 2 & $(3,6 \%)$ \\
PCB8 & 2 & $(3,6 \%)$ \\
PCB9 & 2 & $(3,6 \%)$ \\
Cas isolés & 4 & $(7,1 \%)$ \\
Total hors cas indéterminés & 56 & $(100 \%)$ \\
Cas indéterminés & 15 & \\
(information insuffisante) & & \\
Total général & 71 & \\
\hline
\end{tabular}

adjacentes au couloir, qui obstruent la visibilité. Du fait de la visibilité limitée, l'usager circulant dans le couloir à une vitesse plus élevée ne peut éviter la collision.

Le scénario type PCB3 décrit des accidents dans lesquels un piéton traverse devant un véhicule stationné (véhicule de livraison) dans une voie réservée aux bus, puis est heurté par un véhicule dépassant le véhicule stationné.

Le scénario type PCB4 rassemble des cas où un piéton traverse hors passage piéton et ne perçoit pas un deux-roues motorisé circulant dans une voie réservée aux bus, qui le heurte. Le scénario type PCB5 concerne les cas dans lesquels un véhicule circule en marche arrière dans un couloir de bus (souvent pour réaliser une manœuvre de stationnement) et heurte un piéton traversant ou présent à l'arrière du véhicule. Les autres scénarios types (PCB6 à PCB9) font l'objet d'une brève présentation en annexe.

Les deux-roues motorisés sont impliqués dans la plupart de ces scénarios types (trois cas sur dix-sept dans le scénario type PCB1; quatre cas sur onze dans le scénario type PCB2 ; cinq cas sur six dans le scénario type PCB3 ; la totalité des cas pour le scénario type PCB4 ; aucun cas dans le scénario type PCB5).

La cartographie des cas relevant des trois scénarios types principaux (Fig. 2), montre que les processus d'accidents diffèrent sensiblement selon la façon dont les couloirs de bus sont insérés dans l'espace public. Le scénario type PCB1, par exemple, est presque exclusivement observé dans les rues réservées aux bus ou dans les rues où le couloir de bus est à contresens. Cela est sans doute à relier d'une part au très faible trafic dans les voies bus dans ces configurations, ce qui n'incite pas les piétons à prêter attention à la circulation dans ces voies, et d'autre part, dans certaines situations, au fonctionnement atypique de l'espace (s'agissant des couloirs de bus à contresens), qui

Tableau 7 Description détaillée du scénario type d'accident PCB1

\section{Scénario type PCB1 (décrit sur la base de 17 cas)}

Situation de Un véhicule ${ }^{1}$ circule dans une voie réservée au bus (couloir de bus à contresens dans 6 cas, rue réservée aux bus et conduite piétons dans $7 \mathrm{cas}$ ). Un piéton ${ }^{2}$ marche sur le trottoir adjacent à la voie réservée où circule le véhicule. Il marche le plus souvent dans le même sens que le véhicule $\left(9\right.$ cas). Son attention est souvent mobilisée ${ }^{3}$ par d'autres éléments que la circulation (8 cas). Le piéton est généralement détecté par le conducteur.

Situation Le piéton engage la traversée de la voie sur laquelle circule le véhicule, le plus souvent sans prendre d'information d'accident visuelle, alors que le conducteur, qui n'a pas anticipé cette traversée, poursuit sa route.

Situation Le conducteur effectue le plus souvent une manœuvre d'urgence (10 cas, en général un freinage ou un freinage avec d'urgence un déport latéral).

Situation de Le véhicule heurte le piéton.

choc

${ }^{1}$ Il s'agit d'un bus dans 10 cas et d'un deux-roues motorisé dans 3 cas ; les autres cas concernent respectivement un vélo, un fourgon, un taxi, et un véhicule de police.

${ }^{2}$ Le piéton est un adulte dans les 17 cas de ce scénario type.

${ }^{3}$ L'attention du piéton est focalisée sur des amis (4 cas), souvent de l'autre côté de la rue, ou sur un bus qu'il souhaite prendre, situé sur les voies opposées (1 cas) ; le piéton écoute de la musique ( 2 cas) ou est en discussion au téléphone ( 1 cas). 


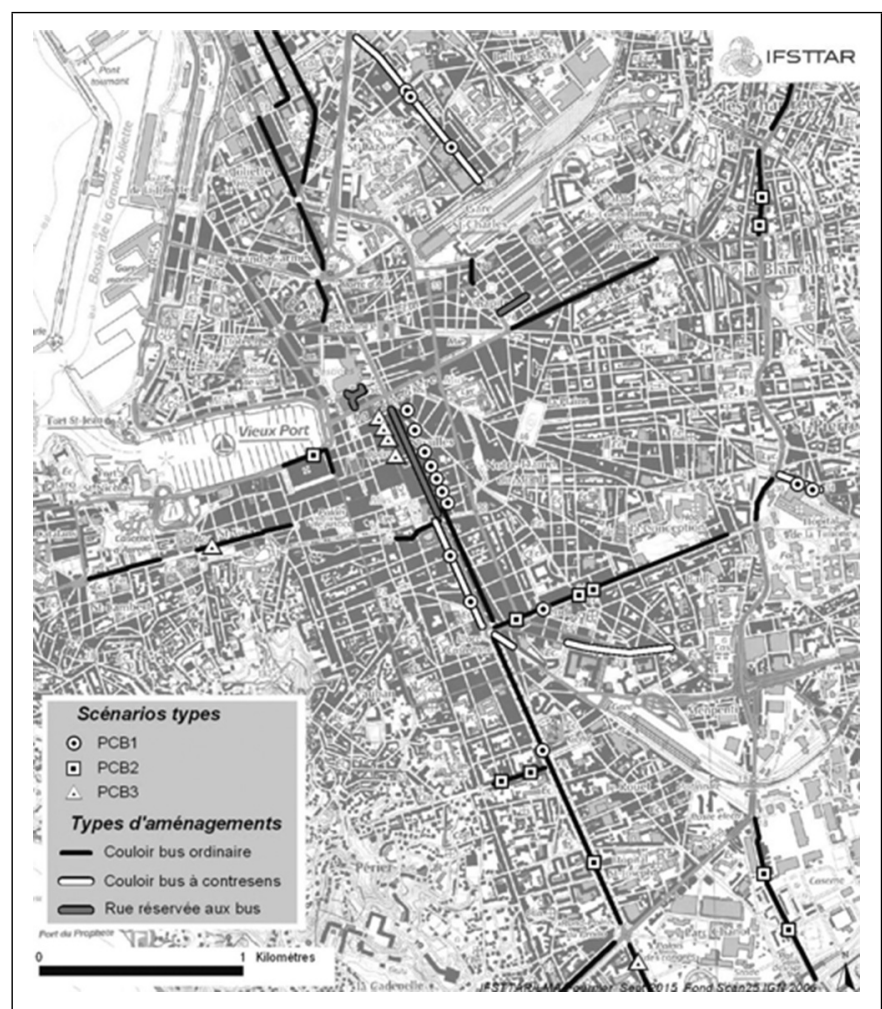

Fig. 2 Répartition spatiale des 34 cas d'accidents relevant des scénarios types d'accidents PCB1, PCB2 et PCB3

Nota : Un accident survenu en dehors des limites de cette carte, relevant du scénario type PCB1 et se déroulant dans un couloir de bus ordinaire, n'est pas représenté.

peut parfois induire les piétons en erreur concernant le sens du trafic dans la voie réservée.

A contrario, les cas relevant du scénario type PCB2 se concentrent exclusivement sur les grands boulevards de la ville où les couloirs de bus sont aménagés dans le sens général. Dans ces rues, la juxtaposition de plusieurs files banalisées et d'une voie bus dans le même sens génère des conflits entre des piétons se faufilant au milieu des véhicules arrêtés et des véhicules circulant plus rapidement dans le couloir de bus. Enfin, le scénario type PCB3 est observé dans les trois configurations d'aménagement des voies réservées, mais il se concentre dans l'hypercentre de la ville où la densité de commerces génère de nombreux arrêts de véhicules de livraison dans les voies réservées aux bus, ces véhicules jouant alors le rôle de masque à la visibilité entre les piétons et le trafic de la voie réservée.

\section{Conclusions et perspectives pour l'aménagement des voies réservées aux bus}

Nous nous sommes intéressés dans cet article aux effets sur la sécurité des piétons des voies réservées aux bus. Les travaux sur ce sujet rapportés dans la littérature scientifique internationale tendent à montrer que ces voies réservées, du moins s'agissant des couloirs de bus ordinaires et des couloirs à contresens, ont un effet plutôt défavorable sur la sécurité des piétons. Ces publications restent cependant relativement peu nombreuses, et portent pour une part sur des contextes assez différents de ceux des villes européennes. Nous avons en conséquence proposé une contribution sur ce sujet, reposant sur l'analyse des phénomènes d'accidents dans les rues de la ville de Marseille équipées de telles voies.

L'évaluation des risques associés aux infrastructures équipées de voies réservées aux bus s'est appuyée sur des analyses comparatives de sections équipées et de sections témoins appariées (à environnement urbain identique, donc avec une fréquentation piétonne comparable, et en prenant en compte les volumes de trafic). Cette méthode a pu être appliquée à l'évaluation des risques d'accident de piéton sur les sections équipées de couloirs de bus aménagés dans le sens de la circulation générale, par rapport à des sections non équipées de couloir réservé. Les résultats présentés font apparaître que le risque pour un véhicule de heurter et blesser un piéton est significativement supérieur (d'environ $67 \%$ ) sur les sections de rues équipées de tels couloirs, par rapport à des sections similaires non équipées. Une tendance comparable (risque accru d'environ $42 \%$ ), mais non significative, est obtenue si l'on s'intéresse au risque, pour un piéton, d'être heurté en traversant de telles sections, par rapport à des sections similaires non équipées de couloir de bus. Ces résultats suggèrent un effet plutôt défavorable de l'équipement des rues en couloirs de bus ordinaires sur la sécurité des piétons, et paraissent en cohérence avec les évaluations antérieures, et notamment les solides études avant-après de Devenport, concernant Londres, et de Chen et al., concernant la ville de New York [5][3].

Afin d'apporter un éclairage sur l'opportunité de l'extension de l'usage des voies réservées aux bus à d'autres catégories (véhicules à fort taux d'occupation, véhicules électriques, etc.), nous avons également comparé le risque de heurter et de blesser un piéton, pour un véhicule circulant dans un couloir de bus (dans le sens de la circulation générale), par rapport à un véhicule circulant dans les voies banalisées adjacentes. Cette analyse montre que les véhicules roulant dans ces couloirs ont un risque significativement supérieur (d'un facteur d'environ 3,5), par rapport à ceux qui empruntent les voies banalisées adjacentes. Ce résultat suggère que l'éventualité de l'ouverture des couloirs de bus à de nouvelles catégories de véhicules ne devrait être envisagée qu'avec une certaine circonspection.

Le risque élevé lié à la circulation dans les couloirs de bus paraît, dans le cas des deux-roues motorisés, en cohérence avec des travaux antérieurs qui ont mis en évidence un sur-risque plus général d'implication de ces usagers dans 
les accidents corporels (de tous types) lorsqu'ils circulent dans les voies réservées aux bus, par rapport aux voies adjacentes [25].

Nous n'avons pas pu procéder aux mêmes analyses quantitatives des risques pour le cas plus rare des rues équipées de couloirs de bus à contresens de la circulation générale et des rues réservées aux bus, du fait d'obstacles méthodologiques (difficulté de trouver des sections témoins appropriées) et de l'absence de données de trafic automobile suffisantes sur une grande partie de ces rues. Cependant, nous avons pu établir que sur les sections équipées de couloirs de bus à contresens, $49 \%$ des accidents de piétons se produisent dans le couloir de bus, malgré un trafic évidemment très restreint par rapport à celui des voies opposées, ouvertes à la circulation générale. Ce résultat, qui n'est pas très éloigné de la proportion de $41 \%$ obtenue par Brownfield et Devenport dans le cas de couloirs à contresens dans la ville de Londres à la fin des années 1980 [6], suggère l'existence d'un risque d'accident de piéton particulièrement élevé dans de tels couloirs. D'ailleurs, sur le sujet plus particulier du risque de collision entre bus et piéton, Vayre met en évidence un risque plus de six fois supérieur dans les couloirs à contresens, par rapport aux autres configurations, dans l'agglomération parisienne [7].

L'étude détaillée des accidents suggère que le sur-risque lié à la circulation dans les couloirs de bus ordinaires tient en partie à la juxtaposition de files banalisées, où le trafic est régulièrement congestionné, et d'un couloir de bus, où le trafic est faible et la vitesse plus élevée. Cette juxtaposition favorise les accidents de piétons traversant entre des véhicules arrêtés puis heurtés par un véhicule non détecté circulant dans le couloir de bus. D'autres travaux, portant sur les accidents de piétons liés aux transports collectifs, ont également mis en évidence le rôle des masques à la visibilité créés par des véhicules en circulation [26]. Parmi les perspectives à étudier concernant la prévention de ces accidents, on pourrait évoquer la possibilité de mise en place d'un terre-plein entre le couloir de bus et les voies banalisées, éloignant légèrement la voie réservée, plus rapide, des voies contiguës, et favorisant une meilleure visibilité mutuelle entre piétons et conducteurs. Mais les inconvénients possibles d'une telle disposition, introduisant une segmentation supplémentaire de l'espace public, seraient cependant à prendre en considération (sur les inconvénients de la complication des espaces publics, voir [27]). En outre, une réduction des vitesses des usagers circulant dans les couloirs, par exemple par la mise en place de déflexions verticales comme des « coussins » ralentisseurs, pourrait également avoir un impact positif.

Dans les couloirs de bus à contresens, la plupart des cas d'accidents impliquent des piétons traversant le couloir sans détecter un véhicule qui y circule, souvent du fait de l'absence de prise d'information visuelle en direction de ce véhicule. Cette absence de prise d'information visuelle $\mathrm{du}$ piéton est à relier à l'organisation atypique de tels espaces, pouvant induire en erreur le piéton au sujet du sens de circulation dans le couloir de bus. La non-détection $\mathrm{du}$ véhicule approchant est également à relier au caractère silencieux des bus contemporains ( 6 des 8 cas étudiés se produisant dans les couloirs de bus à contresens impliquent un bus). Les moyens de prévenir ces accidents sont limités, en dehors de la simple suppression des couloirs de bus à contresens. Brownfield et Devenport recommandent l'usage de signaux sonores et des feux de jours pour les bus [6]. La mise en place de barrières entre trottoir et couloir de bus à contresens fait partie des premières mesures qui ont été mises en œuvre [12], mais les effets de tels équipements restent mal connus.

Enfin, dans les rues réservées aux bus, les accidents impliquant des piétons relèvent principalement de deux scénarios types. Le premier correspond au scénario type observé dans les voies réservées à contresens et implique des piétons traversant sans détecter un véhicule et sans prendre d'information visuelle dans sa direction. Ici, l'absence de prise d'information dans la direction du véhicule heurtant est davantage liée au très faible trafic dans ces rues qu'aux particularités de leur aménagement. Les potentialités d'un éventuel renforcement des contrastes (par le traitement des revêtements, notamment) entre la chaussée où circulent les bus et les espaces réservés aux piétons devraient cependant être étudiées. D'autre part la perspective d'une réduction des vitesses des bus dans les rues fermées au trafic automobile pourrait être examinée : ce type de mesure a été mis en œuvre avec succès concernant la circulation des trams dans des zones piétonnes [28, p. 193]. Le second scénario type implique des piétons traversant devant un véhicule de livraison stationné dans la voie réservée. Cela tient à l'environnement très commerçant des rues dans lesquelles on rencontre ce genre de configuration, et au faible trafic qui incite les véhicules de livraison à stationner sur la chaussée. Pour ce second type d'accident, des évolutions dans la réglementation et l'organisation des livraisons et des pratiques de stationnement correspondantes pourraient vraisemblablement être envisagées.

Globalement, dans l'ensemble des voies réservées aux bus, la part des véhicules non autorisés dans les accidents touchant des piétons est importante (de l'ordre de $60 \%$ ). Cela suggère qu'une meilleure observation des règles d'utilisation des couloirs de bus pourrait être de nature à améliorer la sécurité des piétons. Le contrôle de l'observation de ces règles n'est cependant pas très simple dans la mesure où celles-ci impliquent nécessairement des tolérances (comme l'utilisation d'un couloir pour accéder à une place de stationnement, par exemple). En outre, les risques potentiellement induits par un éventuel renforcement de la répression dans ce domaine sont à prendre en 
considération : un tel renforcement pourrait par exemple avoir pour conséquence d'augmenter la part de deux-roues motorisés circulant sur l'axe de la voie (dans l'espace entre deux files opposées), ce qui pourrait aussi comporter des risques pour la sécurité des piétons [29].

Les résultats présentés souffrent de certaines limites. Concernant l'évaluation des risques, les intervalles de confiance des estimations sont assez larges, du fait que l'échantillon qui a pu être utilisé pour l'analyse reste relativement restreint, et que les effectifs d'accidents considérés sont (heureusement) limités. D'autre part, les tendances mises en évidence ne portent que sur un terrain d'étude particulier. Une extension de l'analyse à d'autres villes permettrait de consolider les résultats, et d'étudier d'autres configurations que celles rencontrées à Marseille (par exemple : voies réservées aux bus en position centrale, couloirs de bus latéraux séparés par des terre-pleins).

Néanmoins, les analyses rapportées ici représentent une contribution originale concernant les liens entre voies réservées aux bus et sécurité des piétons dans le contexte français, où peu de travaux scientifiques ont été publiés. L'originalité du présent travail tient aussi à l'analyse qualitative détaillée de cas d'accidents, qui permet d'accéder à une compréhension des processus d'accidents et d'ouvrir quelques perspectives pour la prévention des accidents. Dans leur ensemble, les résultats présentés sont en cohérence avec les enseignements de la littérature internationale et suggèrent que les couloirs de bus, dans le sens de la circulation ou à contresens, sont plutôt défavorables à la sécurité des piétons. Ils montrent également que la façon dont les voies réservées sont intégrées dans l'espace public conditionne les formes d'accidents de piétons et donc les moyens à mettre en œuvre pour leur prévention.

Remerciements Nous remercions les services de MarseilleProvence-Métropole qui nous ont donné accès à différents recueils de données. Nous remercions également JeanEmmanuel Michel (Ifsttar) pour sa contribution à cette recherche. Ce travail a été en partie réalisé dans le cadre du projet RED, concernant les risques émergents de la mobilité durable, financé par l'Agence nationale de la recherche (ANR).

\section{Références}

1. Organisation de coopération et de développement économique (OCDE) (1969) Améliorations et innovations dans les réseaux d'autobus urbains. OCDE, Paris, $235 \mathrm{p}$

2. Groupement des autorités responsables de transport (GART) (2013) L'année 2012 des transports urbains. GART, Paris, 47 p

3. Chen, L, Chen, C, Ewing, R, McKnight, CE, Srinivasan, R, Roe, $M$ (2013) Safety countermeasures and crash reduction in New
York city-Experience and lessons learned. Accid Anal Prev 50: 312-322

4. Høye, A, Elvik, R, Sørensen, WJ, Vaa, T (2012) Trafikksikkerhetshåndboken. Transportøkonomisk Institutt, Oslo, $821 \mathrm{p}$

5. Devenport, J (1987) An Evaluation of Bus Lane Safety, Report no ATWP80. London Accident Analysis Unit, London, $46 \mathrm{p}$

6. Brownfield, J, Devenport, J (1989) Road Safety Issues for the Design of Bus Priority Schemes, Contractor Report no 180. TRRL, Crowthorne (UK), $126 \mathrm{p}$

7. Vayre, P (2001) Le risque accidentel du piéton dans l'agglomération parisienne. C R Acad Sci Ser III Vie 324 : $1175-1179$

8. Goh, KCK, Currie, G, Sarvi, M, Logan, D (2013) Road safety benefits from bus priority, an empirical study. Transp Res Rec 2352: $41-49$

9. Duduta, N, Adriazola, C, Hidalgo, D, Lindau, L A, Jaff, R (2012) Understanding road safety impact of high-performance bus rapid transit and busway design features. Transp Res Rec 2317: 8-14

10. Bocarejo, JP, Velasquez, JM, Diaz, CA, Tafur, L E (2012) Impact of bus rapid transit systems on road safety. Lessons from Bogotá, Colombia. Transp Res Rec 2317: 1-7.

11. Berg, WD, Smith, RL, Walsh, TW, Notbohm, TN (1981) Evaluation of a contraflow arterial bus lane. Transp Res Rec 798: 45-49

12. Passalacqua, A (2008) Séparer ou périr, conception et pratique du couloir réservé à Paris (1960-1975). Mélanges de l'école française de Rome $120: 59-76$

13. LaPlante, J, Harrington, T (1984) Contraflow bus lanes in Chicago: safety and traffic impacts. Transp Res Rec 957: 80-90

14. Kropidlowski, CR (1988) Chicago's contra-flow and concurrent flow bus lane experience. In: ITE Compendium of Technical Papers. Institute of Transportation Engineers, Washington DC, pp 295-298

15. Brenac, T., Nachtergaële, C., Reigner, H. (2003) Scénarios types d'accidents impliquant des piétons et éléments pour leur prévention. INRETS, Collections de l'INRETS (Rapport no 256), Arcueil, $207 \mathrm{p}$

16. Huguenin-Richard, F (2010) Comportements, tactiques et conduites déviantes des piétons en situation de traversée complexe : le cas lors du franchissement de voies en site propre dans Paris. In: Granié, MA, Auberlet, JM (coords), Le piéton, nouvelles connaissances, nouvelles pratiques et besoins de recherche. INRETS, Collections de l'INRETS (Actes no 127), Bron, pp 91-107

17. Hall, P, Hass-Klau, C (1985) Can Rail Save the City? The Impacts of Rail Rapid Transit and Pedestrianisation on British and German Cities. Gower, Aldershot (UK), $241 \mathrm{p}$

18. Michel, JE, Fournier, JY, Clabaux, N (2013) Place des deux-roues motorisés dans la circulation urbaine : une étude observationnelle. Transp Environ Circ 217: 58-61

19. Elvik, R, Vaa, T (2004) The handbook of road safety measures. Elsevier Science, Amsterdam, $1078 \mathrm{p}$

20. Greenland, S, Robins, JM (1985) Estimation of a common effect parameter from sparse follow-up data. Biometrics 41: 55-68

21. Hasselbald, V, McCrory, DC (1995) Meta-analytic tools for medical decision-making: a practical guide. Med Decis Making 15(1): 81-96

22. DerSimonian, R, Laird, N (1986) Meta-analysis in clinical trials. Control Clin Trials 7: 177-188

23. Brenac, T (1997) L'analyse séquentielle de l'accident de la route. INRETS, Collections de l'INRETS (Outils et méthodes no 3), Arcueil, $79 \mathrm{p}$

24. Brenac, T, Fleury, D (1999) Le concept de scénario type d'accident de la circulation et ses applications. Rech Transp Secur 63 : 63-76.

25. Clabaux, N, Fournier, JY, Michel, JE (2014) Powered two-wheeler drivers' crash risk associated with the use of bus lanes. Accid Anal Prev 71: 306-310 
26. Millot, M, Battaglia, V, Deboudt, B et al. (2015) Étude des accidents piétons sur des rues avec aménagements de sites de transports collectifs, phase 1, rapport d'étude. Cerema DTM, Aix en Provence, $59 \mathrm{p}$

27. Maître, E (2015) Public spaces re-designed for trams in French cities: safety concerns. Adv Transp Stud 37: 119-128

28. Fontaine, L (dir.) (2015) Operation and safety of tramways in interaction with public space: Analysis and outcomes - Detailed report, COST TU1103 Action Final Report, COST Association, Bruxelles, $211 \mathrm{p}$

29. Clabaux, N, Fournier, JY, Michel, JE (2014) Powered two-wheeler drivers' risk of hitting a pedestrian in towns. J Saf Res 51 : 1-5

\section{Annexe}

En complément de la présentation des principaux scénarios types d'accidents de piétons dans les voies réservées aux bus (voir section 6), nous résumons schématiquement ci-après les scénarios types moins représentés :

- PCB6 : Un piéton traverse en confiance sur un passage piéton ; un conducteur circulant dans une voie réservée aux bus détecte trop tardivement ce piéton ou n'anticipe pas sa traversée et le heurte.

- PCB7 : Un jeune piéton, préalablement détecté, s'engage en courant, surprenant le conducteur circulant dans une voie réservée aux bus, qui ne peut l'éviter. Le jeune piéton est souvent accompagné d'autres personnes, et son attention est mobilisée par d'autres éléments que la circulation.

- PCB8 : Un bus heurte avec le rétroviseur un piéton situé sur le trottoir lors d'une manœuvre d'arrêt, à une station de bus.

- PCB9 : Un piéton court pour attraper un bus venant de démarrer et entre en collision avec le flanc du bus.

Certains des scénarios types décrits dans le corps de l'article ou dans cette annexe s'apparentent à des scénarios types plus généraux déjà décrits par ailleurs. Ainsi les scénarios types PCB1, PCB2, PCB5, PCB6 et PCB7 présentent respectivement d'importantes similitudes avec les scénarios types $6,3,15,9$ et 7 de la référence [15]. 